\title{
Investigación y desarrollo en preservación digital: un balance internacional
}

\author{
Por Miquel Térmens
}

\begin{abstract}
Resumen: Se presenta una panorámica de las acciones de investigación y desarrollo en preservación digital en el ámbito internacional, incluyendo un apartado dedicado a la situación en España. La mayor atención se da en las aplicaciones de preservación digital en bibliotecas y archivos, pero también se presenta someramente la situación en otros entornos como los repositorios institucionales, los archivos administrativos, los archivos personales, la industria aeroespacial y los museos. El análisis de los protagonistas de la investigación lleva a destacar el papel preeminente de algunas bibliotecas y archivos nacionales, de determinados países y una incipiente entrada de empresas de software. En cuanto a las líneas de investigación destaca la integración de herramientas para generar sistemas de preservación integrados.
\end{abstract}

Palabras clave: Preservación digital, Bibliotecas digitales, Archivos electrónicos, Administración electrónica, Digitalización.

\section{Title: Research and development in digital preservation: an internatio- nal review}

Abstract: The article presents a survey of international research studies and development in digital preservation, devoting a section to the situation in Spain. The author outlines applications in areas such as institutional repositories, administrative records, personal archives, aerospace industry and museum archives, but the greatest attention is given to digital preservation applications in libraries and archives. The pre-eminent role of certain libraries and the national archives and records of certain countries is evident in an analysis of the protagonists of this research, as well as the emerging role of software companies. The major lines of research include the integration of tools to create integrated preservation systems.

Keywords: Digital preservation, Digital libraries, Digital archives, Electronic administration, Digital imaging, Digital curation.

Térmens, Miquel. "Investigación y desarrollo en preservación digital: un balance internacional". El profesional de la información, 2009, noviembre-diciembre, v. 18, n. 6, pp. 613-624.

DOI: 10.3145/epi.2009.nov.04

\section{Antecedentes}

Desde mediados de la década de 1990 distintas instituciones y empresas están estudiando el problema de la preservación futura de los documentos digitales. La expansión de este tipo de documentos ha llevado a que en determinados ámbitos su presencia sea mayoritaria y por tanto se convierta en ineludible su conservación por razones patrimoniales, culturales, jurídicas o de otro tipo.

La preservación digital es una tarea no sólo de archiveros y bibliotecarios sino de todos los actores relacionados con la creación, gestión y uso de cada documento digital. La preservación digital supone, en relación con la conservación de los documentos en papel, un importante reto tecnológico, pero también de otros tipos: legal (¿se pueden descompilar los programas in- formáticos para facilitar su conservación?), económico (¿quién financiará las actuaciones de preservación?), organizativo (¿de quién es la responsabilidad de cada acción? ¿cómo se asegura la continuidad de las decisiones?) (Keefer; Gallart, 2007).

Centrándonos en los aspectos técnicos, las principales estrategias de preservación utilizadas son la recopia, la migración de formatos y la emulación en plataformas diferentes a la original, de sistemas y software (Rothenberg, 1999; Serra-Serra, 2001; SerraSerra, 2008). A diferencia de los documentos tradicionales (impresos, fotografías), en los documentos digitales debe distinguirse entre el soporte físico de los datos (disco magnético, disco óptico, etc.) y los ficheros propiamente dichos, creados según unos formatos y normas concretos. Ante la continua obsolescencia de los soportes no cabe otra solución que la migración de 
los datos hacia soportes más actuales. En cuanto a los ficheros, aún no existe un consenso científico respecto a la alternativa entre las técnicas de migración y emulación; los distintos proyectos de investigación o de aplicación se decantan por una u otra según sus propios objetivos, testeando las ventajas e inconvenientes de cada alternativa.

\section{"Los mayores avances se concentran en algunos países europeos, en los EUA y en el núcleo de Oceanía formado por Australia y Nueva Zelanda"}

A medio camino entre los aspectos técnicos y los organizativos, se encuentra la elaboración, aún no concluida, de aplicaciones tipo checklist que ayuden a los técnicos y gestores a tomar las mejores decisiones de preservación ante un tipo determinado de documentos. También se han de destacar los avances en la creación de estándares internacionales de metadatos descriptivos y de gestión que han de acompañar los documentos para facilitar su tratamiento y uso futuros.

Todo este panorama compuesto por avances en diferentes sectores quizá no tendría la coherencia global que está alcanzando si no existiera un consenso sobre el modelo general a seguir. En 2002 se publicó bajo el impulso de la NASA el Open Archival Information System Reference Model (OAIS) (Reference, 2002). El modelo OAIS, totalmente aceptado internacionalmente y estandarizado como norma ISO 14721, establece las seis funciones que han de configurar un sistema integral de preservación, así como los flujos de gestión de los ficheros y sus metadatos. La primera función, quizá en la que más se está trabajando en la actualidad, es la de entrada de ficheros en el sistema de preservación, pues necesita aplicaciones y metodologías automatizables y replicables que se han de adaptar a las distintas situaciones documentales reales. Tanto la sistematización conceptual relacionada con la preservación digital, como el software que se ha empezado a implementar, orbitan alrededor de este modelo consolidado. Proyectos de referencia como Planets o Caspar se basan eminentemente en la elaboración y aplicación del modelo OAIS.

Presentar una panorámica coherente de la investigación en preservación digital es difícil. El principal obstáculo estriba en la dificultad de separar lo que es investigación pura de las actuaciones de elaboración o de simple implementación de soluciones; en la actualidad sólo hay soluciones integrales, llave en mano, para grandes organizaciones tipo archivo o biblioteca nacional, pero en el resto de situaciones se dispone de aplicaciones parciales que, en el mejor de los casos, deben personalizarse e integrarse en los sistemas de información corporativos. Aún así, estas adaptaciones o elaboraciones parciales son de interés general pues aún pocas instituciones o empresas se atreven a llevarlas a cabo.

Se presenta en primer lugar un recorrido por las principales iniciativas de investigación en el ámbito internacional y en el ámbito español, sobre todo centradas en el marco de actuación de bibliotecas y archivos. A continuación se muestran algunos campos específicos de estudio en la preservación digital.

\section{Panorama internacional}

La investigación en preservación digital hasta el momento ha estado liderada a escala internacional por algunas bibliotecas y archivos nacionales y por proyectos con la participación de universidades, empresas de software e instituciones depositarias de documentación. Los avances más importantes están bastante concentrados geográficamente en algunos países europeos, en los Estados Unidos y en el núcleo de Oceanía formado por Australia y Nueva Zelanda.

En el ámbito paneuropeo, la Comisión Europea está promoviendo la investigación en este área por medio de las convocatorias del quinto, sexto y séptimo Programa Marco, que han dado lugar a los proyectos Erpanet, Digital Preservation Europe (DPE), Planets, Caspar, Protage, Shaman, KEEP, PrestoPRIME y LiWA. Estos proyectos, a menudo interconectados, están implementando aplicaciones de validación de formatos, emuladores de ordenadores personales, procedimientos de auditoria de repositorios y sistemas expertos de planificación de preservación. La Comisión Europea también está promocionando el avance de los archivos electrónicos y de los estándares que los harán viables. En la primera línea se sitúa DLM Forum, un marco de cooperación entre archiveros que se concreta en la convocatoria periódica de un congreso europeo (el último en Toulouse en 2008). Como fruto de este interés, se elaboraron las normas MoReq, ahora en su segunda edición conocida como MoReq2 (2008), que marcan los requerimientos que han de cumplir los sistemas de gestión de archivos electrónicos, entre los que se encuentran los relacionados con la preservación de los documentos.

Los Países Bajos son uno de los núcleos más antiguos y activos de investigación y desarrollo en preservación digital gracias a las actividades de su Biblioteca nacional (Koninklijke Bibliotheek) y Archivo nacional (Nationaal Archief); las dos instituciones participan, de una forma u otra, en la mayoría de los grandes pro- 


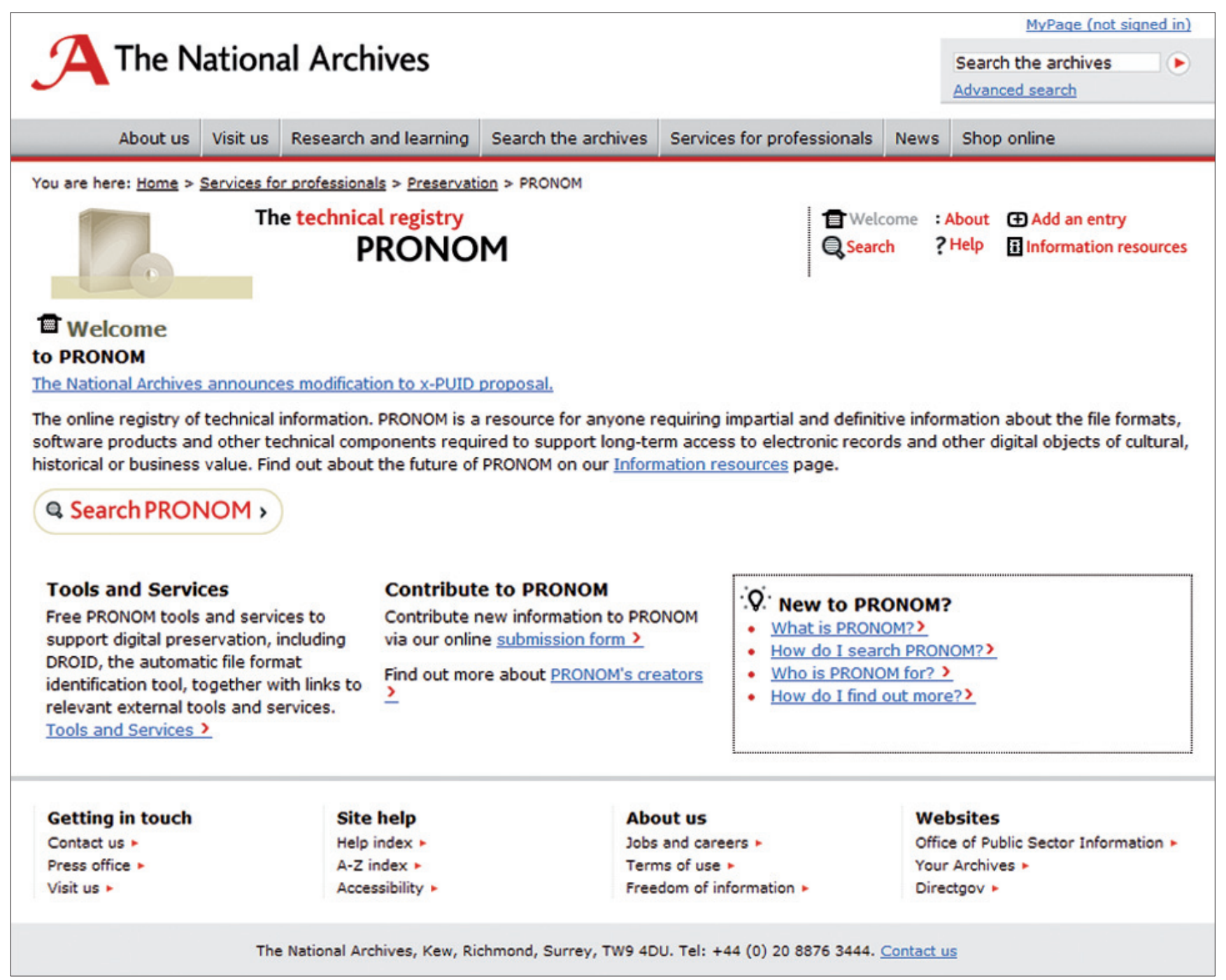

Pronom es el sistema de identificación de formatos creado por los archivos nacionales del Reino Unido. http://www.nationalarchives.gov.uk/pronom/

yectos europeos. La Biblioteca nacional, con la ayuda tecnológica de $I B M$, ha montado el e-Depot, un repositorio diseñado específicamente para preservar documentación digital científica y cultural (Hoorens el al., 2007); también han elaborado el programa emulador Dioscuri (Van-der-Hoeven; Lohman; Verdegem, 2007), integrado dentro del proyecto Planets.

\section{"Los Países Bajos son uno de los núcleos más antiguos y activos de investigación en preservación digital gracias a las actividades de su biblioteca nacional y archivo nacional"}

En el Reino Unido se está viviendo una importante eclosión de proyectos gracias a la financiación y al impulso del Joint Information Systems Committee (JISC)
(Beagrie et al., 2008), de la British Library (destacamos el proyecto Digital Lives), de The National Archives (TNA) (proyecto Digital Continuity, entre otros) y la creación de servicios como el Digital Curation Centre $(D C C)$ de Edimburgo. Por la trascendencia y el reconocimiento que ha recibido a escala mundial, sobresale la creación por TNA del registro de formatos Pronom, el identificador PUID y la aplicación de verificación de formatos Droid.

En Francia, tanto la Bibliothèque National con el Système SPAR (Bermes et al., 2008) como los Archives Nationales con el proyectoPil@e están trabajando para la preservación de sus repositorios y archivos digitales. SPAR es un sistema de almacenamiento para la biblioteca digital Gallica, para el archivo del web francés y para otros servicios de la Bibliothèque $\mathrm{Na}$ tional. ConPil@e, aún en elaboración, los ministerios contarán con un sistema centralizado de preservación digital de los documentos administrativos.

Portugal es reconocido por los proyectos llevados a cabo por la Biblioteca Nacional de Lisboa, por la Di- 
recção Geral de Arquivos (repositorio RODA) y por la Universidade do Minho (servicios de migración CRiB) (Ramalho et al., 2008).

En Alemania se están efectuando diversas implementaciones. Por un lado la Biblioteca nacional (Deutche Nationalbibliothek), la biblioteca universitaria de Goettingen (Niedersaechsische Staats-und Universitatetsbibliothek) e IBM emprendieron el proyecto Kopal, de hecho una adaptación del e-Depot holandés a un entorno de bibliotecas digitales en red. El gobierno federal también ha impulsado por medio del Physikalisch-Technische Bundesanstalt (PTB) la creación de un archivo digital seguro y certificado a disposición de la administración federal (Siegfried, 2005): se trata del proyecto Archisafe, que entró en servicio a finales de 2008. Por último, el catálogo de criterios de Nestor es un buen ejemplo de checklist para evaluar y certificar depósitos seguros de preservación (Dobratz; Schoger; Strathmann, 2007).

En Estados Unidos se ha de destacar el papel impulsor de la Library of Congress como coordinadora del National Digital Information Infrastructure and Preservation Program (NDIIPP), plan en el que se consagró la visión de preservación en red. La misma Library of Congress ha ayudado a llevar a la práctica y es depositaria de nuevos sistemas de metadatos como MODS (metadatos descriptivos), METS (metadatos técnicos) y Premis (metadatos de preservación). También es crucial el papel de los archivos nacionales National Archives and Record Administration (NARA), con la elaboración de un sistema de archivo electrónico (ERA). La Stanford University creó en 2001 el sistema Lockss que mediante una red cerrada peer to peer permite la replicación de datos científicos y su preservación (Seadle, 2006). La University of North Carolina at Chapel Hill (UNC) y la University of California, San Diego (UCSD) están elaborando el sistema iRODS que, de forma similar al anterior, utiliza los recursos de las redes GRID para asegurar la preservación de grandes volúmenes de datos. UCSD y San Diego Supercomputer Center (SDSC) lideran un consorcio que ha creado Chronopolis, un sistema ya operativo de almacenamiento redundante de grandes volúmenes de datos (Hutt et al., 2008). También se ha de mencionar la Cornell University, que ha indagado en aspectos de gestión y sostenibilidad de los sistemas de preservación, así como el Florida Center for Library Automation (FCLA), que ha elaborado Daitss, un sistema integral de archivo de preservación (Caplan, 2004), y la Harvard University con la elaboración de la aplicación de verificación de formatos Jhove (Abrams, 2007) y la propuesta de Global Digital Format Registry (GDFR), recientemente transformada en Unified Digital Formats Registry (UDFR).
En el ámbito privado destaca el papel de la fundación Internet Archive no sólo en la preservación de la Web mundial sino también en la creación de herramientas de libre uso para su aplicación en otros contextos (el crawler Heritrix y los buscadores WERA y Wayback) y en el soporte a iniciativas nacionales como Minerva de la Library of Congress (Day, 2003). También es un caso de éxito Portico, otra fundación privada que desde enero de 2006 tiene en explotación el primer servicio disponible en el mercado para la preservación de revistas electrónicas (Fenton, 2006).

Australia y Nueva Zelanda son núcleos muy potentes en este campo (National Archives of Australia, 2004). Nueva Zelanda está apostando de forma importante por la creación de repositorios abiertos basados en descripciones Dublin Core (DC) e interrogables con el protocolo OAI-PMH; han puesto en práctica un sistema para extraer los metadatos de los documentos: The National Library of New Zealand (NLNZ) Metadata Extraction Tool. La National Library of Australia ha impulsado la preservación de la Web a escala nacional $(P A D I)$ y también internacional con la creación de la red International Internet Preservation Consortium (IIPC); los archivos nacionales (National Archives of Australia) han elaborado distintas aplicaciones de software para sistemas de preservación, como Xena, un validador y normalizador de formatos. Sin salir de Australia, es preeminente la iniciativa VERS, para la gestión de los archivos electrónicos del gobierno del estado de Victoria, que culminó con la apertura en 2005 del PROV Digital Archive.

\section{Situación en España}

Hasta no hace mucho tiempo España ha contado con un nivel bajo de investigación en preservación digital, excepto en sistemas de gestión documental relacionados con la administración electrónica. Una notable excepción en el campo de la investigación fue la lectura en 2005 de la tesis doctoral de Bárbara Muñoz-de-Solano (Muñoz-de-Solano-y-Palacios, 2005); posteriormente, en el año 2007 dos grupos españoles lograron incorporarse por primera vez en proyectos competitivos europeos de preservación digital: se trata de Easy Innova, una spinoff de la Universitat de Girona, que participa en el proyecto Protage, y la consultora privada Inmark que coordina el proyecto Shaman. Asimismo, la Biblioteca Nacional de España forma parte del grupo de trabajo Long Term Preservation (LPT), en el que también participan las bibliotecas nacionales de Alemania, Austria, Gran Bretaña, Noruega, Portugal, República Checa y Suiza, cuya finalidad es examinar el panorama general de la preservación digital a largo plazo existente en las bibliotecas nacionales europeas y encontrar una solución viable de implantación a nivel internacional. 


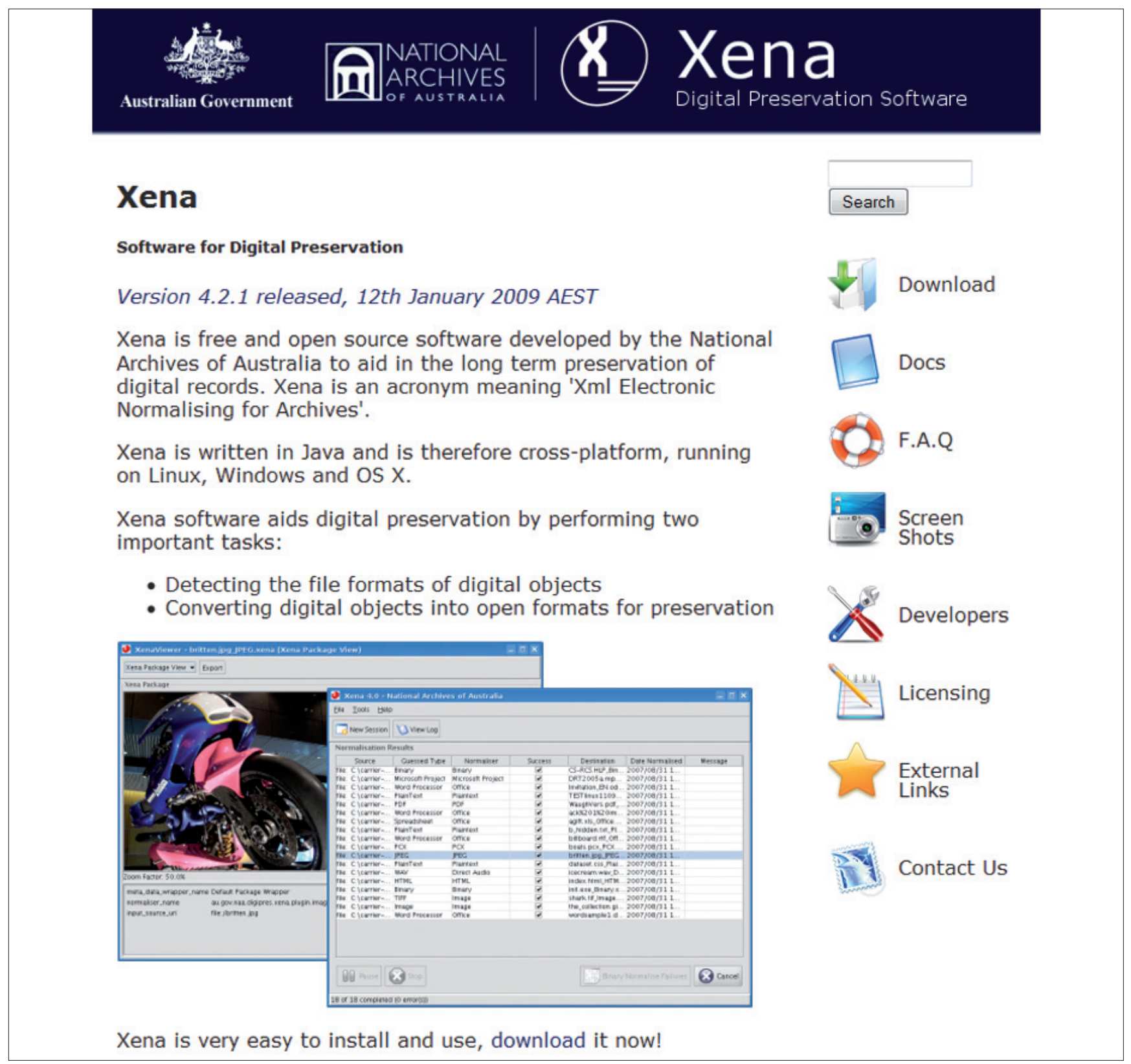

Xena es el software libre de caracterización y normalización de ficheros creado por los archivos nacionales de Australia. http://xena.sourceforge.net

\section{"Debemos constatar un cierto retraso de las instituciones españolas en este campo"}

En el ámbito operativo y aún en fase de expansión se ha de mencionar el proyecto de preservación de la Web Padicat, de la Biblioteca de Catalunya, equiparable a los mejores existentes en el mundo y que ha sido presentado como modelo por el propio IIPC (Llueca, 2006).

Desde el Ministerio de Cultura cabe destacar la creación en abril de 2007 de la Comisión española sobre la digitalización y la accesibilidad en línea del material cultural y la conservación digital (Cedalmac), encargada de llevar a cabo en España las acciones contenidas en la Recomendación de la Comisión Europea de 24 de agosto de 2006 sobre la digitalización y la accesibilidad en línea del material cultural y la conservación digital. La preservación así mismo se está abordando dentro de las actividades del Grupo de Trabajo de Patrimonio Digital del Ministerio de Cultura y las comunidades autónomas, pero no se conocen resultados tangibles de estos dos grupos de trabajo. La Biblioteca Nacional de España ha integrado la preservación como actividad de soporte a su política de digitalización de materiales. Se ha de reseñar en su haber la traducción al español de la obra de Priscilla Caplan Entender PREMIS (Caplan, 2009), que ha de facilitar la aplicación de este esquema de metadatos en el mundo hispánico. 
Rebiun (Red de Bibliotecas Universitarias Españolas) ha creado recientemente un grupo de trabajo con el fin de impulsar la aplicación de soluciones de preservación en las bibliotecas universitarias y, de forma más específica, para preservar los repositorios abiertos y las bibliotecas digitales. Muestra de este interés fue la celebración del VIII Workshop Rebiun sobre Proyectos Digitales, dedicado a La preservación digital: memoria de futuro (Murcia, 20 y 21 de octubre de 2008).

La gestión documental ligada a la administración electrónica presenta más resultados. Desde hace unos años el Consejo Superior de Administración Electrónica, del Ministerio de Administraciones Públicas, está impulsando la modernización de la administración española, en especial la del Estado. Podemos destacar la elaboración de protocolos de seguridad (como Magerit, Metodología de Análisis y Gestión de Riesgos de los Sistemas de Información), donde tiene un papel destacado el CNN-CERT (Equipo de respuesta a incidentes de seguridad de la información del Centro Criptológico Nacional).

La Ley 11/2007, de 22 de junio, de acceso electrónico de los ciudadanos a los servicios públicos (BOE 23-6-2007) impone que a partir del 1 de enero de 2010 las distintas administraciones españolas deberán estar preparadas para aceptar y gestionar los documentos digitales que los ciudadanos les remitan en el ejercicio de sus derechos. Esta ley supondrá la puesta en práctica de la administración electrónica, lo que implica plantearse los sistemas necesarios para preservar la documentación digital que gestionarán las administraciones públicas. Las distintas administraciones, no sólo la central, están adaptando sus normativas y elaborando sistemas que permitan afrontar el nuevo reto.

Dentro de la Generalitat de Catalunya se han realizado estudios sobre los esquemas de metadatos (Grup de Treball d'Innovació Tecnològica de la Subdirecció General d'Arxius) y se ha implementado (ahora en fase de pruebas) un repositorio seguro de documentación electrónica para el conjunto de las administraciones públicas catalanas (iArxiu, de la Agencia Catalana de Certificación (CatCert)). En 2008 la Asociación de Archiveros de Catalunya (AAC) decidió integrarse dentro del proyecto internacional Interpares 3, coordinado por la University of British Columbia (Canadá), aportando el análisis de distintas problemáticas existentes en archivos de la administración universitaria y local (Duranti; Preston, 2008).

Otras iniciativas a destacar son las que en este momento lleva a cabo el Gobierno Vasco a fin de preservar la Web y de implantar la administración electrónica, y los trabajos sobre gestión documental realizados en el Archivo Municipal de Cartagena (Delgado-Gómez, 2008).

\section{Otras áreas de actuación}

Además de los proyectos citados que se ocupan especialmente de grandes bibliotecas y archivos, la preservación digital ha abordado otras áreas de actuación con problemáticas específicas. Son relevantes las investigaciones que se ocupan de los repositorios open access y la preservación ligada a los archivos administrativos con las implicaciones legales que comporta, que aún no se ha desplegado pero que por exigencia legal se espera que tenga una gran evolución próximamente. Finalmente se citan proyectos específicos que han buscado respuestas a necesidades particulares que exigen pequeñas variaciones respecto a las soluciones genéricas.

\subsection{La preservación de los repositorios institucio- nales}

En los últimos años se ha vivido una gran expansión de los repositorios institucionales de documentación científica (tesis, preprints, informes, etc.), dentro del movimiento open access. En estos momentos crece la preocupación en bibliotecas y universidades por la salvaguarda de unos repositorios y bibliotecas digitales que cada vez acumulan más documentación y que empiezan a ejercer un papel central en la comunicación científica internacional (Keefer, 2005; Verheul, 2006; Wheatley, 2004). Por tanto, ahora se demanda que estos repositorios no sólo sean usables y visibles, sino también seguros (a ser posible con certificación) y con políticas que aseguren su preservación a largo plazo (Trustworthy Repositories, 2007).

En ese punto se ha de recordar que la mayoría de los repositorios institucionales no pueden ser considerados como repositorios de preservación pues, según indica Premis (Premis, 2008), un repositorio de preservación es "un repositorio que bien como responsabilidad única o como parte de múltiples responsabilidades desempeña la preservación a largo plazo de los objetos digitales bajo su custodia". DSpace y Fedora, dos de los softwares más utilizados en los repositorios institucionales, ya están orientados a facilitar las acciones de preservación en la ingestión, importación y transformación de metadatos, migración de formatos e implantación de un plan de vigilancia tecnológica (Jantz, 2006). La integración desde mayo de 2009 de estos dos softwares en una única organización, DuraSpace, favorecerá aún más su evolución, como ya lo indica el inicio de las pruebas de DuraCloud, un sistema de almacenamiento y preservación de objetos digitales que utilizará los servicios de computación y replicación en la nube. También se están elaborando aplicaciones de preservación aplicadas al software ePrints, en este caso a cargo de los proyectos británicos Preserv y KeepIt.

Otras actuaciones (por ejemplo la aplicación Plato (Becker, 2007) del proyecto Planets) van en la línea 


\section{ExLibris Rosetta}

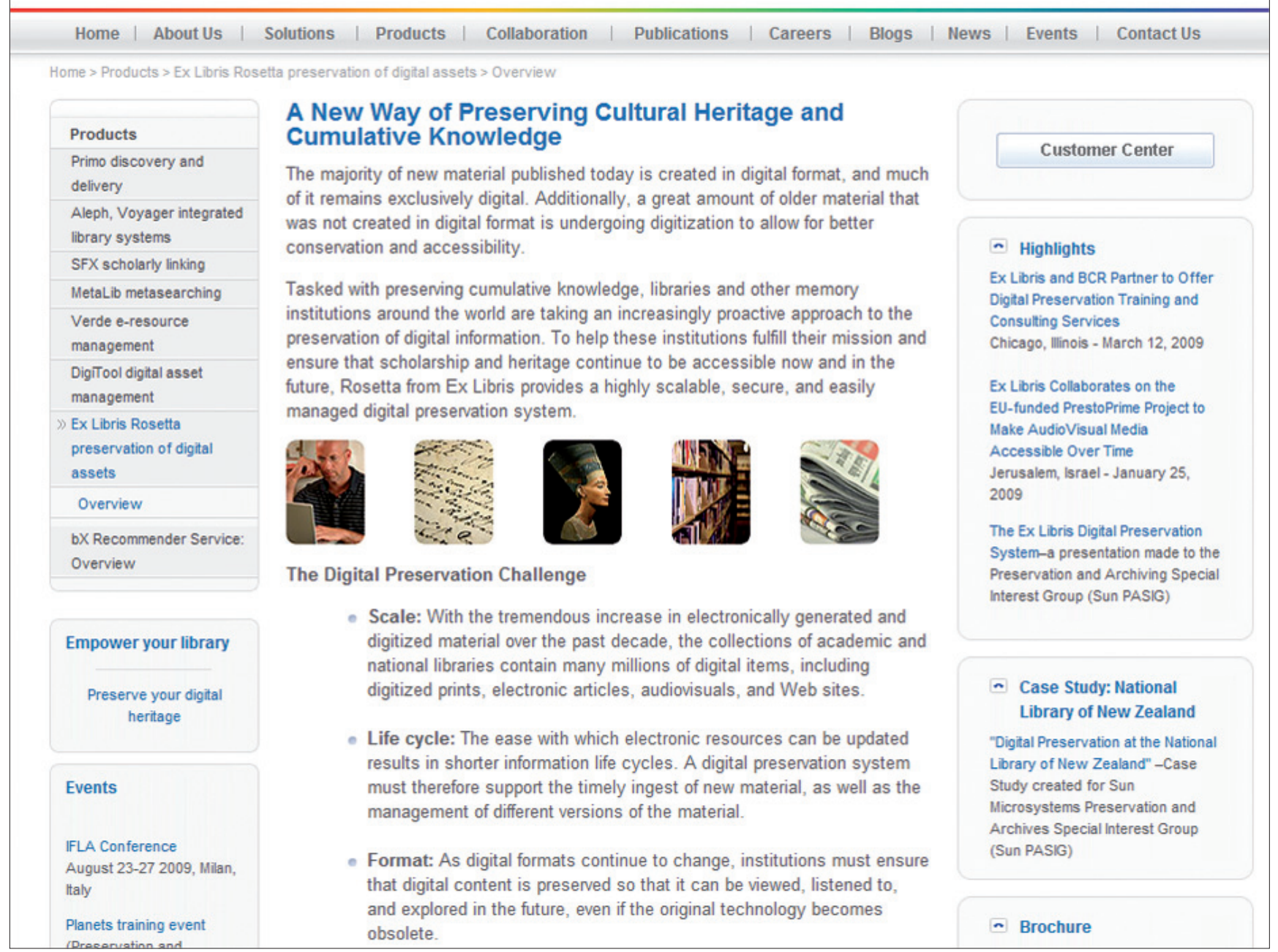

Rosetta, de ExLibris, es un software comercial de preservación integral basado en la experiencia de la Biblioteca nacional de Nueva Zelanda. http://www.exlibrisgroup.com/category/ExLibrisRosettaOverview

de crear procedimientos de auditoria (bajo la forma de checklist $\mathrm{u}$ otras) que faciliten a los gestores de preservación la toma de las decisiones técnicas más apropiadas.

\subsection{La preservación de los archivos administrati- vos}

Hay que destacar que en la preservación de los archivos administrativos los aspectos de autenticidad y mantenimiento de la integridad de los documentos son fundamentales. Así en los archivos administrativos, sean de la administración pública o bien de empresas privadas, no sólo se han de preservar los documentos sino también las firmas electrónicas y las marcas de tiempo (data stamp) que les otorgan autenticidad y los ubican cronológicamente.

La línea de investigación y desarrollo más activa en este sector es la encaminada a la creación de depósitos seguros y certificados de documentación administrativa, en especial la de carácter público. Van en esta línea los sistemas alemán Archisafe, el australiano VERS y el español iArxiu. En el ámbito organizativo, se han de destacar los desarrollos realizados a favor de la aplicación normalizada de metadatos siguiendo la norma ISO 23081:2006 Metadata for Records and Records Management Processes.

\subsection{La preservación en otros sectores}

El Cidoc (The International Committee for $\mathrm{Mu}$ seum Documentation), uno de los comités del ICOM (International Council of Museums) está coordinando la investigación para hallar soluciones de preservación para distintas problemáticas propias de los museos, en especial la preservación del Net-Art y la permanencia de los sistemas virtuales, como pueden ser las reconstrucciones virtuales de monumentos del pasado.

Las empresas de construcción aeronáutica están trabajando de forma conjunta en el ámbito europeo (AeroSpace and Defence Industries Association of Europe, $A S D-S T A N$, con el liderazgo del consorcio Airbus) y de 
los Estados Unidos (Aerospace Industries Association, $A I A$ ) para consolidar formatos, procedimientos y sistemas que aseguren la permanencia de toda la documentación técnica de los aviones construidos al menos durante el tiempo de vida comercial de los mismos. En el caso europeo se están elaborando los estándares Lotar.

\section{"Desde la empresa privada se constata un interés creciente en este campo, cuyo dominio bien pronto tendrá un carácter estratégico"}

Asimismo se está trabajando en pos de la preservación en distintas áreas científicas en las que se están elaborando grandes bases de datos: genética, biología, recursos marinos, meteorología, astronomía, etc. En este caso los mayores problemas se refieren al gran volumen de los datos a conservar (medibles en terabytes y petabytes), a la configuración en red de la mayoría de estas bases de datos y cómo asegurar su preservación sin afectar a su uso directo por los investigadores. Destaca el trabajo de la Agencia Espacial Europea (ESA) en la preservación de los datos obtenidos por satélite (European, 2008).

La preservación de los archivos personales está recibiendo una especial atención en el Reino Unido, donde se están llevando a cabo proyectos por parte de TNA y de bibliotecas depositarias de fondos políticos, como la Bodleian Library, en Oxford, depositaria de los archivos del partido conservador (Workbook, 2007). Los problemas que más preocupan y se están investigando son: la recuperación de información almacenada en soportes obsoletos (en especial disquetes), la migración de los formatos originales obsoletos a otros actuales y de preservación (por ejemplo de Wordstar a Open Office) y la generación automatizada de metadatos descriptivos para documentos que fueron creados sin ellos.

\section{Los protagonistas de la investigación}

Hasta este punto hemos seguido una relación detallada, aunque no exhaustiva, de las actuaciones más destacadas en preservación digital. Ante esta acumulación de datos corremos el riesgo de no poder identificar correctamente cuáles son los actores principales en este campo y hacia donde están encaminando su trabajo. A continuación intentaremos clarificar estos aspectos.

Hasta hace bien poco se podía considerar que era un lujo investigar y aún más realizar avances en preservación digital. La expansión de la Web y con ella la de toda la información digital ha obligado a la mayoría de las instituciones públicas y empresas a centrarse en la ingente tarea de redefinir sus procesos internos de trabajo y de creación de sistemas de interacción con los documentos digitales, dentro de los cuales las webs y las intranets son la parte más visible. En este contexto sólo las entidades más fuertes en tamaño, responsabilidades y recursos económicos han podido pensar en el futuro de los documentos digitales sin menoscabo de la actividad diaria.

Por un lado destacan las entidades y los investigadores que en la segunda mitad de la década de 1990 investigaron y normalizaron las técnicas de digitalización retrospectiva de documentos, estableciendo entonces los formatos y los detalles técnicos más apropiados para escanear fotografías, libros o documentos de archivo, y ahora, quince años más tarde, en una evolución natural, se ocupan del reto de preservar los ficheros que ayudaron a crear: Library of Congress, Cornell University y $N A R A$, entre otros. Así mismo se constata que los centros que de una forma más temprana se lanzaron a crear repositorios digitales son los que después también han sido pioneros en la preservación, como Library of Congress (National Digital Library Program en 1994), y Koninklijke Bibliotheek (Depot for the Dutch Electronic Publications, DNEP, en 1996).

Las bibliotecas y archivos nacionales, así como algunas grandes bibliotecas universitarias son otro de los focos de avance; destacan en esta lista la British Library y The National Archives, en el Reino Unido, y las bibliotecas y archivos nacionales de Australia, Nueva Zelanda, Alemania y los países nórdicos. Tampoco podemos olvidarnos de los esfuerzos que se realizan en Alemania por parte de universidades y organismos del gobierno federal.

Desde la empresa privada se constata un interés creciente en este campo, cuyo dominio bien pronto tendrá un carácter estratégico, pero dados los riesgos tecnológicos que estos desarrollos aún presentan, las empresas se están incorporando al mercado de la mano de instituciones públicas que actúan de banco de pruebas. El interés privado no sólo se constata entre las empresas centradas en la automatización de sistemas de gestión de la documentación, sino también en el conjunto de las empresas de informática, así como entre las de defensa y seguridad. Entre las primeras podemos observar la reciente entrada de Ex Libris (a partir del aprendizaje de la National Library of New Zealand), entre las segundas IBM (socio de la biblioteca nacional de los Países Bajos, Koninklijke Bibliotheek) y Tessella (contratista de The National Archives), y entre las terceras Lockheed Martin Corporation (contratista de los archivos nacionales de los Estados Unidos) y Harris Corporation (contratista de US Government Printing Office). 


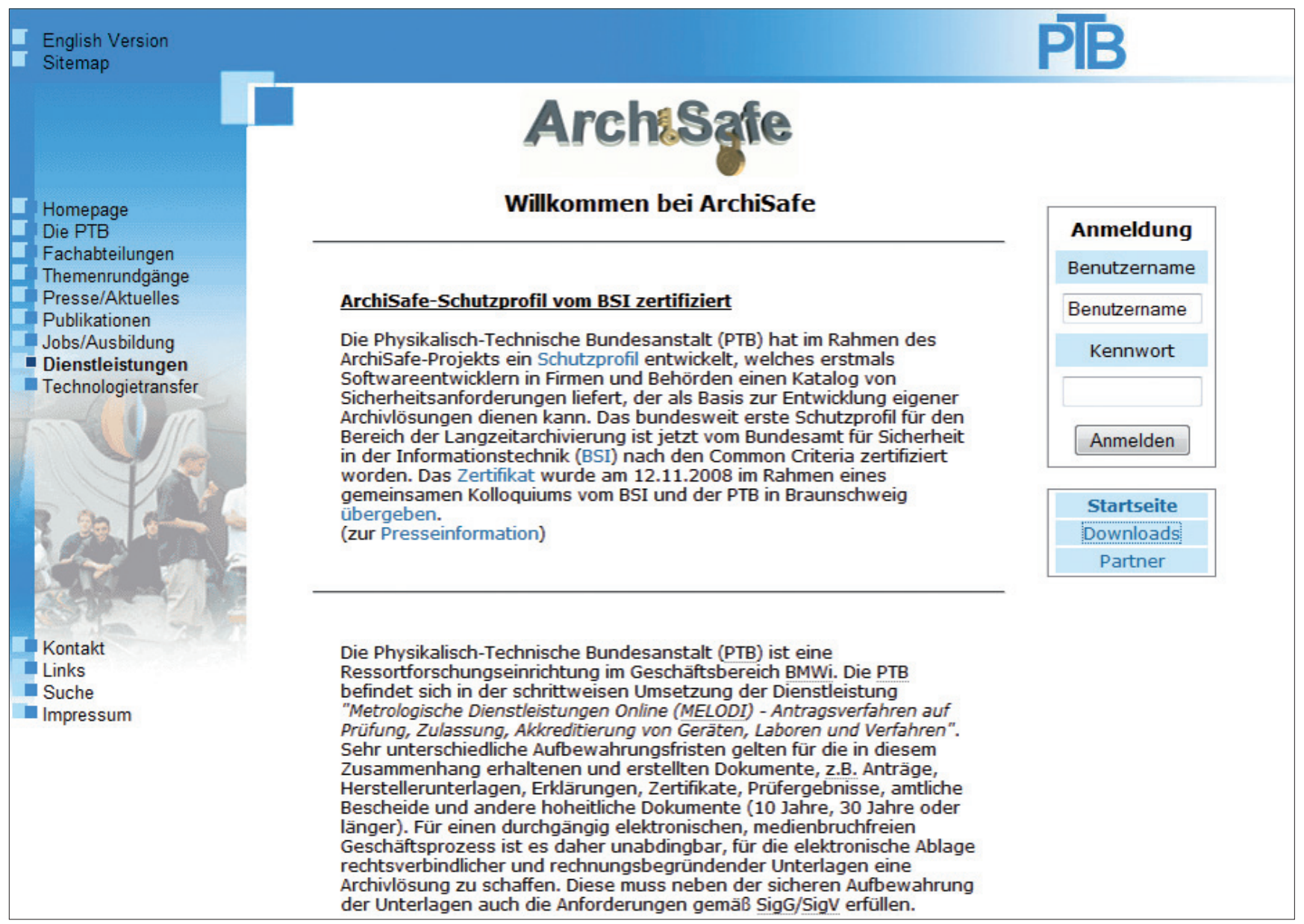

Archisafe, del Instituto Nacional de Metrología (PTB) de Alemania, uno de los pocos depósitos seguros de preservación actualmente en funcionamiento. http://www. archisafe.de

\section{Las principales líneas de investigación}

Una revisión de los proyectos en curso permite concluir que se ha entrado en una segunda fase en la evolución de los sistemas de preservación. Los aspectos más teóricos que a principios de esta década acaparaban la atención ahora están dejando paso a creaciones más prácticas y aplicables en entornos reales. Así, los debates sobre las alternativas técnicas de preservación (migración, emulación, encapsulamiento, ordenador universal virtual (UVC), etc.), sobre los formatos más recomendables o sobre el modelo funcional, sin estar cerrados, tienen un menor peso relativo frente a los esfuerzos en pos de la integración de herramientas, la automatización de los procesos de ingestión o la creación de sistemas expertos de ayuda a la toma de decisiones en preservación.

En el primer ámbito "clásico" descubrimos que las acciones de migración de formatos bien pronto serán guiadas por sistemas pseudo expertos como Planets Testbed; asimismo la emulación ha dejado de ser una propuesta quimérica para convertirse en una realidad cotidiana en los centros de datos con software de virtualización del tipo VMware, Microsoft Virtual PC y Sun Virtual Box, hecho que está respaldando la elabora- ción de productos específicamente orientados a la preservación como Dioscuri.

\section{"Destaca la integración de aplicaciones para generar sistemas de preservación completos"}

Entre las "nuevas" líneas de actuación destaca la integración de aplicaciones para generar sistemas de preservación completos; tienen esta orientación Planets Interoperability Framework y el software Caspar, ambos aún en elaboración, sistemas ya en uso como Daitss, e-Depot, Irods, Kopal, PROV Digital Archive, $S P A R$ y VERS, y los sistemas comerciales DIAS, de IBM, Safety Deposit Box, de Tessella, y Rosetta, de Ex Libris. Otra línea con buenos resultados es la centrada en generar sistemas de ayuda a la toma de decisiones o de auditoria de sistemas, como Check-up, Drambora y Plato.

En el caso de la preservación de las revistas científicas electrónicas los avances han sido enormes debido a la preocupación tanto de editores como de clientes 
ante la posibilidad de pérdida de estos contenidos. En la actualidad ya se encuentran operativos sistemas alternativos de preservación al menos para las revistas publicadas por los editores grandes y medianos $(e-D e-$ pot, Portico, Clockss), quedando pendientes en especial las soluciones para la preservación de las revistas open access (Day, 2008; Kenney et al., 2006). Las urgencias se han trasladado recientemente hacia la preservación de los libros, que han sido objeto de grandes proyectos de digitalización y para los que aún no se han diseñado unas políticas de preservación adaptadas al elevado volumen de estos depósitos (Rieger, 2008).

Aunque este artículo se ha centrado en los aspectos tecnológicos de la preservación digital, no puede concluir sin recordar que los aspectos organizativos, económicos y legales también están recibiendo una gran atención; son una muestra el actual interés por los costes y la sostenibilidad económica de la preservación (Davies et al., 2007; Blue Ribbon Task Force, 2008).

\section{Conclusiones}

La investigación en preservación digital es un campo emergente y por ello no perfectamente definido. Una prueba de su carácter novedoso se encuentra en el aún bajo nivel de implementación práctica de sus resultados: sólo algunas grandes instituciones y empresas han empezado a aplicar, a menudo de forma parcial, sistemas de preservación digital que, por otro lado, aún no se están comercializando de forma amplia.

También hemos comprobado que las iniciativas, sean de tipo experimental o ya aplicado, están apareciendo cada vez en más países e instituciones de todo tipo. El conocimiento sobre preservación digital es cada vez mayor y está aumentando su difusión; recordemos en este sentido que buena parte del software específico de preservación que se está elaborando es de código abierto, basado en xml y Java y por tanto, perfectamente adaptable a la mayoría de los entornos de trabajo.

Por último debemos constatar un cierto retraso de las instituciones españolas en este campo, que quizá cabe achacar al previo retraso con el que las bibliotecas, los archivos y los museos del país entraron en las tareas de automatización, primero, y en los servicios digitales, después. Es previsible que en un futuro cercano la situación cambie debido a las propias exigencias de los servicios digitales que se han implementado en los últimos años. Este cambio es inevitable en el caso de las bibliotecas universitarias, cada vez con repositorios digitales de mayor volumen que habrá que preservar, y también en los archivos de la administración, implicados en los nuevos roles de gestión y custodia de los documentos electrónicos que marca la Ley $11 / 2007$.

\section{Bibliografía}

Abrams, Stephen. "Automated characterization in preservation workflows". En: Tools and Trends: International Conference on Digital Preservation at the occasion of the retirement of Johan Steenbakkers. The Hague: Koninklijke Bibliotheek, National Library of the Netherlands, 2007.

http://www.kb.nl/hrd/congressen/toolstrends/presentations/Abrams.pdf

Beagrie, Neil. National digital preservation initiatives: an overview of developments in Australia, France, the Netherlands, and the United Kingdom and of related international activity. Washington DC: Council on Library and Information Resources, 2003.

http://www.clir.org/pubs/reports/pub116/pub116.pdf

Beagrie, Neil; Semple, Najla; Williams, Peter; Wright, Richard. Digital preservation policies study. JISC, 2008.

http://www.jisc.ac.uk/publications/publications/jiscpolicyfinalreport.aspx

Becker, Christoph. "Evaluating preservation strategies: decision support for preservation planning". En: Tools and Trends: International Conference on Digital Preservation at the occasion of the retirement of Johan Steenbakkers. The Hague: Koninklijke Bibliotheek, National Library of the Netherlands, 2007.

http://www.kb.nl/hrd/congressen/toolstrends/presentations/Becker.pdf

Bermes, Emmanuelle; Carbone, Isabelle-Dussert; Ledoux, Thomas; Lupovici, Christian. "La préservation numérique à la Bibliothèque nationale de France: présentation technique et organisationnelle". En: IFLA Congress 2008, 2008.

http://www.ifla.org/IV/ifla74/papers/084-Bermes_Carbone_Ledoux_ Lupovici-trans-fr.pdf

Blue Ribbon Task Force on Sustainable Digital Preservation and Access. Sustaining the Digital Investment: Issues and Challenges of Economically Sustainable Digital Preservation. 2008.

http://www.sdsc.edu/News\%20Items/PR121608_brtf_report.html

Caplan, Priscilla. Entender PREMIS. Ministerio de Cultura, 2009. http://www.mcu.es/bibliotecas/MC/PREMIS/Presentacion.html

Caplan, Priscilla. "The Florida Digital Archive and Daitss: a working preservation repository based on format migration". International journal on digital libraries, 2004, v. 6, n. 4, pp. 305-311.

http://www.fcla.edu/digitalArchive/pdfs/IJDL_article.pdf

Davies, Richard; Ayris, Paul; McLeod, Rory; Shenton, Helen; Wheatley, Paul. "How much does it cost? The LIFE Project". LIBER Quarterly, 2007, v. 17 , n. 3-4.

Day, Michael. "Preserving the outputs of scholarly communication for the long-term: a review of recent developments in digital preservation for electronic journal content". En: Jones, Wayne (ed.): E-journals access and management. New York: Routledge, 2008, pp. 39-64.

http://www.ukoln.ac.uk/preservation/publications/2008/e-journals/draftv01.pdf

Day, Michael. Collecting and preserving the World Wide Web: a feasibility study undertaken for the JISC and Wellcome Trust. UKOLN, University of Bath, 2003.

http://library.wellcome.ac.uk/assets/wtl039229.pdf

Delgado-Gómez, Alejandro. "Modelo conceptual para el desarrollo de un sistema de conservación a largo plazo de bases de datos del Ayuntamiento de Cartagena". Tabula, 2008, n. 11, pp. 291-300.

Dobratz, Susanne; Schoger, Astrid; Strathmann, Stefan: "The nestor catalogue of criteria for trusted digital repository evaluation and certification". JoDI: Journal of digital information, 2007, v. 8, n. 2.

http://journals.tdl.org/jodi/article/view/199/180

Duranti, Luciana; Preston, Randy (eds). International research on permanent authentic records in electronic systems (InterPARES) 2: experiential, interactive and dynamic records. Rome: Associazione Nazionale Archivistica Italiana, 2008.

http://www.interpares.org/display_file.cfm?doc=ip2_book_complete.pdf

European long term data preservation common policy draft version 1. GSCB (Ground Segment Coordination Body) Long Term Data Preservation Working Group, 2008.

http://earth.esa.int/gscb/ltdp/EuropeanLTDPCommonPolicy_DraftV1.pdf 
Fenton, Eileen-Gifford. "An overview of Portico: an electronic archiving service". Serials review, 2006, v. 32, n. 2, pp. 81-86.

Hoorens, Stijn; Rothenberg, Jeff; Van-Orange-Nassau, Constantijn; Van-der-Mandele, Martijn, Levitt, Ruth. Addressing the uncertain future of preserving the past. Towards a robust strategy for digital archiving and preservation. Santa Monica (EUA): RAND Corporation, 2007.

http://www.rand.org/pubs/technical_reports/2007/RAND_TR510.pdf

Hutt, Arwen; Westbrook, Brad; Kozbial, Ardys; McDonald, Robert Sutton, Don. "Developing preservation metadata for use in grid-based preservation systems". En: Proceedings of the Fifth International Conference on Preservation of Digital Objects (iPRES 2008), 2008, pp. 145-150.

http://www.bl.uk/ipres2008/ipres2008-proceedings.pdf

Jantz, Ron. "Fedora preservation services - a working group report". En: Long-term Repositories: Taking the Shock out of the Future. APSR, 2006. http://www.apsr.edu.au/longterm/jantz_trusted.ppt

Keefer, Alice C. "Preservación digital y depósitos institucionales" El profesional de la información, 2005, v. 14, n. 6, pp. 404-406.

http://www.elprofesionaldelainformacion.com/contenidos/2005/ noviembre/1.pdf

Keefer, Alice C.; Gallart, Núria. La preservación de recursos digitales: el reto para las bibliotecas del siglo XXI. Barcelona: UOC, 2007.

Kenney, Anne R.; Entlich, Richard; Hirtle, Peter B.; McGovern, Nancy Y.; Buckley, Ellie L. E-journal archiving metes and bounds: a survey of the landscape. Washington, DC: Council on Library and Infomaton Resources, 2006

http://www.clir.org/pubs/reports/pub138/pub138.pdf

Llueca, Ciro. "Archivando la Web, el proyecto Padicat (Patrimonio Digital de Cataluña)". El profesional de la información, 2006, v. 15, n. 6, pp. 473 478.

http://www.elprofesionaldelainformacion.com/contenidos/2006/ noviembre/09.pdf

MoReq2. Model Requirements for the management of electronic records. Update and extensión 2008. Luxembourg: Office for Official Publications of the European Communities, 2008.

http://www.moreq2.eu/

Muñoz-de-Solano-y-Palacios, Bárbara. Fundamentos conceptuales de la preservación del documento digital. Madrid, Universidad Complutense de Madrid, 2005. Tesis doctoral.

National Archives of Australia. digital recordkeeping. Guidelines for creating, managing and preserving digital records. National Archives of Australia, 2004

http://naa.gov.au/Images/Digital-recordkeeping-guidelines_tcm2-920.pdf

PREMIS Data dictionary for preservation metadata. Version 2.0. 2008. 217 p. http://www.loc.gov/standards/premis/v2/premis-2-0.pdf

Ramalho, José-Carlos; Ferreira, Miguel; Faria, Luis; Castro, Rui; Barbedo, Francisco; Corujo, Luis. "RODA and CriB. A service-oriented digital repository". En: Proceedings of the Fifth International Conference on Preservation of Digital Objects (iPRES 2008), 2008, pp. 235-241. http://www.bl.uk/ipres2008/ipres2008-proceedings.pdf

Reference model for an open archival information system (OAIS). Blue Book. Consultative Committee for Space Data Systems, 2002. http://public.ccsds.org/publications/archive/650x0b1.pdf

Rieger, Oya Y. Preservation in the age of large-scale digitization. Washington DC: Council on Library and Information Resources, 2008. http://www.clir.org/pubs/reports/pub141/pub141.pdf

Rothenberg, Jeff. Avoiding technological quicksand: finding a viable technical foundation for digital preservation. Washington DC: Council on Library and Information Resources, 1999.

http://www.clir.org/pubs/reports/rothenberg/pub77.pdf

Seadle, Michael. "A social model for archiving digital serials: LOCKSS". Serials Review, 2006, v. 32, n. 2, pp. 73-77.

Serra-Serra, Jordi. "Gestión de los documentos digitales: estrategias para su conservación”. El profesional de la información, 2001, v. 10, n. 9, pp. 4-18.

http://www.elprofesionaldelainformacion.com/contenidos/2001/ septiembre/l.pdf
Serra-Serra, Jordi. Los documentos electrónicos. Qué son y cómo se tratan. Gijón: Trea, 2008.

Siegfried, Hackel. "The ArchiSafe Project - legally secure and scalable long-term record keeping". En: DLM Forum 2005, 2005.

http://ec.europa.eu/transparency/archival_policy/dlm_forum/doc/26_ hackel_06-10-05pm.pdf

Trustworthy Repositories Audit \& Certification (TRAC): Criteria and Checklist. Version 1.0. February 2007. Chicago: CRL, OCLC, 2007.

http://www.crl.edu/archiving-preservation/digital-archives/metricsassessing-and-certifying/trac-ch

Van-der-Hoeven, Jeffrey; Lohman, Bram; Verdegem, Remco. "Emulation for digital preservation in practice: the results". International journal of digital curation, 2007. v. 2, n. 2.

http://www.ijdc.net/index.php/ijdc/article/view/50/35

Verheul, Ingeborg. Networking for digital preservation. Current practice in 15 National Libraries. München: Saur, 2006.

http://www.ifla.org/V/pr/saur119.htm

Wheatley, Paul. Institutional repositories in the context of digital preservation. Digital Preservation Coalition, 2004

http://www.dpconline.org/docs/DPCTWf4word.pdf

Workbook on Digital Private Papers. Oxford: Bodleian Library, 2007. http://www.paradigm.ac.uk/workbook/index.html

\section{Instituciones, productos y proyectos citados}

Archisafe.

http://www.archisafe.de

Check-up. National Archives of Australia.

http://www.naa.gov.au/records-management/check-up/index.aspx

Clockks.

http://www.clockss.org/

Conversion and Recommendation of Digital Object Formats (CRiB)

http://crib.dsi.uminho.pt/

Cornell University Libraries.

http://www.library.cornell.edu/iris/dpo/

Cultural, Artistic and Scientific knowledge for Preservation, Access and Retrieval (Caspar)

http://www.casparpreserves.eu/

Dark Archive in the Sunshine State (Daitss). http://daitss.fcla.edu/

Digital Continuity. The National Archives.

http://www.nationalarchives.gov.uk/electronicrecords/digitalcontinuity/ default.htm

Digital Curation Centre (DCC).

http://www.dcc.ac.uk/

Digital Information Archiving System (DIAS). IBM.

http://www-05.ibm.com/nl/dias/

Digital Lives.

http://www.bl.uk/digital-lives/index.html

Digital Preservation Coalition (DPC).

http://www.dpconline.org/

Digital Preservation Europe (DPE).

http://www.digitalpreservationeurope.eu

Dioscuri.

http://dioscuri.sourceforge.net

Drambora.

http://www.repositoryaudit.eu/

Droid.

http://droid.sourceforge.net

DSpace.

http://www.dspace.org/ 
DuraCloud.

http://duraspace.org/duracloud.php

DuraSpace.

http://duraspace.org/

Easy Innova.

http://www.easyinnova.com/

Erpanet.

http://www.erpanet.org/

E-depot. Koninklijke Bibliotheek.

http://www.kb.nl/hrd/dd/index.htm

Fedora.

http://www.fedora-commons.org/

Global Digital Format Registry (GDFR).

http://www.gdfr.info/

iArxiu.

http://www.catcert.cat/web/cas/1_4_9_iArxiu.jsp

Inmark.

http://www.inmark.es/

International Internet Preservation Consortium (IIPC).

http://www.netpreserve.org

Internet Archive.

http://www.archive.org

Interpares.

http://www.interpares.org/

iRODS.

https://www.irods.org

Joint Information Systems Committee (JISC).

http://www.jisc.ac.uk/

JSTOR/Harvard object validation environment (Jhove).

http://hul.harvard.edu/jhove/

KEEP.

http://www.keep-project.eu/

KeepIt.

http://preservation.eprints.org/keepit/

Kopal.

http://kopal.langzeitarchivierung.del

LiWA.

http://www.liwa-project.eu/

LOng Term Archiving (Lotar)

http://www.prostep.org/en/project-groups/long-term-archiving-lotar.html

Metadata Encoding and Transmission Standard (METS).

http://www.loc.gov/standards/mets

Metodología de Análisis y Gestión de Riesgos de los Sistemas de Información (Magerit).

http://www.csae.map.es/csi/pg 5m20.htm

Metadata Object Description Standard (MODS).

http://www.loc.gov/standards/mods

Minerva: Library of Congress Web Archive.

http://lcweb2.loc.gov/diglib/lcwa/html/lcwa-home.html

Nationaal Archief

http://www.nationaalarchief.nl/

National Archives and Records Administration (NARA). Electronic Records Archives. (ERA)

http://www.archives.gov/era/

National Archives of Australia.

http://www.naa.gov.au/
National Digital Information Infrastructure and Preservation Program. http://www.digitalpreservation.gov/

National Library of New Zealand (NLNZ) Metadata Extraction Tool. http://meta-extractor.sourceforge.net/

PADI.

http://www.nla.gov.au/padi/index.html

Pandora.

http://pandora.nla.gov.au/index.html

Patrimoni digital de Catalunya (Padicat).

http://www.padicat.cat/es/index.php

Planets.

http://www.planets-project.eu

Plato.

http://www.ifs.tuwien.ac.at/dp/plato/intro.html

Portico.

http://www.portico.org/

Premis.

http://www.loc.gov/standards/premis/

Preserv.

http://preserv.eprints.org/

PReservation Organizations using Tools in AGent Environments (Protage). http://www.protage.eu/

PrestoPRIME.

http://www.prestoprime.org/

Pronom.

http://www.nationalarchives.gov.uk/pronom/

Repositório de Objectos Digitais Autênticos (RODA).

http://portal.roda.dgarq.gov.pt/

Rossetta. Ex Libris.

http://www.exlibrisgroup.com/category/ExLibrisRosettaOverview

Safety Deposit Box (SDB). Tessella.

http://www.tessella.com/solutions-technologies/solutions/archiving-digitalpreservation/

Sustaining Heritage Access through Multivalent ArchiviNg (Shaman). http://shaman-ip.eu/

Système de Préservation et d'Archivage Réparti (SPAR).

http://www.bnf.fr/pages/zNavigat/frame/infopro.htm?ancre=numerisation/ num_spar.htm

The International Committee for Museum Documentation. Le Comité International pour la Documentation des Musées (Cidoc).

http://cidoc.mediahost.org/

The National Archives (TNA).

http://www.nationalarchives.gov.uk/

Unified Digital Formats Registry (UDFR)

http://www.gdfr.info/udfr.html

US Government Printing Office (GPO).

http://www.gpo.gov/

Victorian Electronic Records Strategy (VERS).

http://www.prov.vic.gov.au/vers/

Xena.

http://xena.sourceforge.net

Miquel Térmens. Universitat de Barcelona, Departamento de Biblioteconomia i Documentació. Melcior de Palau, 140. 08014 Barcelona termens@ub.edu 